\title{
Alterations in the processing of non-drug-related affective stimuli in abstinent heroin addicts
}

\author{
Zhao-Xin Wang ${ }^{\mathrm{a}}$, John X. Zhang ${ }^{\mathrm{b}, *}$, Qiu-Lin $\mathrm{Wu}^{\mathrm{a}}$, Ning Liu ${ }^{\mathrm{c}}$, Xiao-Ping $\mathrm{Hu}^{\mathrm{d}}$, \\ Raymond C.K. Chan ${ }^{\mathrm{e}}$, Zhuang-Wei Xiao ${ }^{\mathrm{a}, *}$ \\ a Guangdong Key Lab of Medical Molecular Imaging, the First Affiliated Hospital, Shantou University Medical College, Shantou, China \\ ${ }^{\mathrm{b}}$ Department of Psychology, the Chinese University of Hong Kong, Shatin, Hong Kong \\ c Kunming Institute of Zoology, the Chinese Academy of Sciences, Kunming, China \\ d Department of Biomedical Engineering, Georgia Institute of Technology and Emory University, Atlanta, GA, USA \\ e Institute of Psychology, the Chinese Academy of Sciences, Beijing, China
}

\section{A R T I C L E I N F O}

\section{Article history:}

Received 24 September 2008

Revised 19 July 2009

Accepted 10 August 2009

Available online 14 August 2009

\section{Keywords:}

Addiction

Heroin

Affective processing

Emotion

Amygdala

Visual cortex

\begin{abstract}
A B S T R A C T
Long-term exposure to drug may alter the neural system associated with affective processing, as evidenced by both clinical observations and behavioral data documenting dysfunctions in emotional experiences and processing in drug addicts. Although many imaging studies examined neural responses to drug or drugrelated cues in addicts, there have been few studies explicitly designed to reveal their neural abnormalities in processing non-drug-related natural affective materials. The present study asked abstinent heroin addicts and normal controls to passively view standardized affective pictures of positive, negative, or neutral valence and compared their brain activities with functional MRI. Compared to normal controls, addicts showed reduced activation in right amygdala in response to the affective pictures, consistent with previous reports of blunted subjective experience for affective stimuli in addicts. Furthermore, in two visual cortical areas BA 19 and 37 , while the controls showed greater responses to positive pictures than to negative ones replicating literature findings, the addicts showed the opposite pattern. The results reveal a complex pattern of altered processing of non-drug-related affective materials in addicts showing both heightened and blunted neural responses in different brain regions and for different stimulus valence. The present study highlights the importance of brain imaging research on drug addicts' processing of affective stimuli in understanding disruptions in their emotion circuitry.
\end{abstract}

(c) 2009 Elsevier Inc. All rights reserved.

\section{Introduction}

Clinical observations have identified many emotion-related signs in the development and maintenance of drug addiction. For example, substance use is associated with euphoria, and abstinence from drugs produces aversive withdrawal symptoms (e.g., Baker et al., 2004). Theoretically, researchers have also considered emotion as a key element in drug addiction. For example, Baker et al. (2004) argue that the escape and avoidance of negative affect, manifested as specific emotions such as anxiety, irritability, or sadness, is the prepotent motive for addictive drug use. Volkow and Fowler (2000) and Baler and Volkow (2006) propose to associate the intense craving and highly compulsive behaviors in addicts with dysfunctions of the

\footnotetext{
* Corresponding authors. Z.-W. Xiao is to be contacted at Guangdong Key Lab of Medical Molecular Imaging, Shantou University Medical College, Shantou, 515041, China. J.X. Zhang is to be contacted at Department of Psychology, the Chinese University of Hong Kong. Fax: +86754 8612318.

E-mail addresses: jxzhang@cuhk.edu.hk (J.X. Zhang), xiaozw@vip.163.com (Z.-W. Xiao).
}

striato-thalamo-orbitofrontal circuit, which is central to emotion regulation and behavior inhibition. A critical implication from these considerations is that long-term exposure to drug may not only cause neural adaptation to drug and drug-related stimuli but also alter the neural system associated with affective processing of natural reinforcers (Baker et al., 2004; Baler and Volkow, 2006).

One line of research on drug craving, particularly those studies with brain imaging techniques, have documented much evidence that addicts show exaggerated neural responses to drug or drug-related cues (e.g., Garavan et al., 2000; George et al., 2001; Grant et al., 1996). However, imaging studies directly addressing another major aspect of the issue, i.e., how addiction alters responses to non-drug-related, naturally rewarding stimuli including everyday affective stimuli, have been limited. Some studies show addicts to be hypersexual or hypersensitive to money rewards (Washton and Stone-Washton, 1993; Bechara and Damasio, 2002; Bjork et al., 2008) but others show the opposite results (Garavan et al., 2000; Goldstein et al., 2007). With everyday affective stimuli, Gerra et al. (2003) found reduced physiological and neuroendocrine responses in abstinent heroin users, suggesting dysfunctions in their emotion processing. 
Behaviorally, Dawkins et al. (2007) showed that abstinent smokers rated positive clips as less pleasant than did satiated smoker who smoked as usual. de Arcos et al. (2005) reported a tendency of sensitization in cocaine users who rated unpleasant pictures as more unpleasant and pleasant ones as more pleasant. In comparison, patients using alcohol showed a different tendency towards neutral evaluations, i.e., flattening in emotional experience. With explicit motivations and the adoption of standardized affective stimuli, de Arcos and colleagues $(2005,2008)$ provide some of the clearest and most direct behavioral evidence for altered emotional processing and experience in drug addicts, manifested as blunted or sensitized responses to pleasant and unpleasant stimuli. Still, the available data are inconsistent and present a complex pattern where such alterations seem to be modulated by various factors (e.g., type of drug used, nature of the stimuli). This apparently calls for more systematic empirical investigation, particularly brain imaging studies, before more serious attempt of theoretical integration. Wexler et al. (2001) applied brain imaging on addicts with everyday affective stimuli. However, that study was designed to examine craving with drugrelated clips and only included clips eliciting happy or sad emotions for secondary interest.

In line with the de Arcos et al. $(2005,2008)$ studies, the present study utilized functional magnetic resonance imaging (fMRI) to examine brain responses in heroin addicts to reveal how they differ from normal controls in processing non-drug-related affective stimuli. Abstinent addicts were studied as deficits present in abstinent periods, unconfounded by acute withdrawal symptoms, should reflect long-lasting or even permanent alterations to brain circuitry. For ease of comparison with literature studies, the stimuli were selected from the well-known International Affective Picture System (IAPS) and the study focused on the important valence dimension (Russell and Barrett, 1999).

To avoid effects from strategic or reflective processes, participants were asked to perform a passive picture viewing task inside the scanner without reporting or managing their emotional experience. The task, though simple, has been shown to reliably engage emotional processing in previous imaging studies (e.g., Compton et al., 2003; Xiao et al., 2006). Brain regions of interest were those that have been implicated in previous studies of addiction and affect, including the limbic system, hippocampus, amygdala, orbital-frontal cortex, insular, and anterior cingulate. Considering the simplicity and passive nature of the task, activation in areas involved more with regulation or control in emotional processing such as the dorsal lateral prefrontal cortex was not expected.

\section{Methods}

\section{Participants}

Seventeen abstinent heroin addicts $(N=17$, aged between 22.6 and 42.2 years, mean $\pm S D=31.2 \pm 5.4$ years, all male) and 16 heroinnaïve healthy volunteer controls ( $N=16$, aged between 21.4 and 31.8 years, mean $\pm S D=25.2 \pm 3.1$ years, all male) participated in the experiment. All were right-handed native Chinese with normal vision. The controls were recruited from local community, free from any lifetime history of drug dependence.

The patients were recruited from a local clinic where they participated in a mandatory detoxification program in a hospitallike setting but isolated from the society to prevent drug access. Upon entering the clinic, the addicts would be treated by sublingual buprenorphine (4-8 mg/day) for 5 days for detoxification. After the detoxification, they would stay in the clinic for 1 year. They would then be either released from the program or stay for an extended period of time up to one more year. All patients were recruited in the present experiment 2 to 5 months after detoxification when they were no longer in acute withdrawal or taking any medication to control withdrawal. They were active drug users before entering the clinic so the time since detoxification was also their pre-test abstinence time.

For the patients, the inclusion criteria were as follows: (1) a DSMIV diagnosis of heroin dependence within the past 6 months using the Structured Clinical Interview for DSM-IV; (2) a minimum of 6 months self-reported continuous heroin use; (3) normal intelligence; and (4) an ability to provide written informed consent and to comply with the study procedures. The diagnosis of heroin dependence was conducted by a professional psychiatrist when the patients first entered the clinic.

All patients reported heroin use only and were heavy, long-term heroin addicts (range for dose of daily drug use between 0.1 and $3 \mathrm{~g}$, mean $\pm S D=0.72 \pm 0.73 \mathrm{~g}$; mean range for history of heroin use between 2 and 15 years, mean $\pm S D=6.9 \pm 2.9$ years; range for pretest abstinence time between 2 and 5 months, mean $\pm \mathrm{SD}=3.4 \pm$ 0.9 months). All 17 used the "chasing the dragon" method and seven also used intravenous injection.

All participants underwent a pre-test screening concerning drug use history, tobacco and alcohol use, and general health conditions by filling out a questionnaire. All participants except three controls reported daily tobacco consumption (range for daily tobacco usage between 0.5 and 3 packages for patients and between 0 and 1.5 packages for controls, mean $\pm S D=1.2 \pm 0.8$ packages for patients and $0.8 \pm 0.6$ packages for controls). Two patients and three controls reported regular alcohol use while the rest reported none, minimal, to infrequent use but none had any history of alcohol dependence, which was checked before the experiment, based on the DSM-IV diagnosis standard for alcohol dependence, by one of the authors who is a clinician. For both groups, potential participants would be excluded if they had any history of active neurological or serious psychiatric disorders or HIV-positive status (except heroin dependence for the patients).

Observing the Declaration of Helsinki, written informed consent was obtained from each participant following a research protocol approved by the Human Subjects Review Committee of the First Affiliated Hospital of Shantou University Medical College. All participants particularly the patients were clearly informed that they were free to participate and to terminate throughout the study without incurring any punishment. Each participant would receive 30 RMB (about US \$4) as compensation for their participation.

\section{Stimuli}

The stimuli consisted of 80 affective pictures and 20 neutral pictures, all selected from the International Affective Picture System (IAPS) developed by Lang et al. (1998a) and widely used in emotion studies (e.g., de Arcos et al., 2005; Gerra et al., 2003). Half of the affective pictures were of positive valence and the other half of negative valence, with their mean normative arousal ratings matched (5.1 for the positive pictures, 5.6 for the negative pictures, two-sample $t$-test $p>0.1$ ).

\section{Procedure}

Participants lay supine inside the MRI scanner, wearing earphones and goggles specially designed for the MR environment (Resonance Technology Company, Inc., Los Angeles, CA) and instructed to keep their head still. Their heads were further restrained with padding behind the neck and between the head and the head coil. The visual stimuli were presented through the goggles controlled with a PC computer using the Inquisit software (Millisecond Inc., Seattle, WA). An eye-tracking system that came with the stimulus presentation system was used to monitor eye movement to ensure fixation on the stimuli during scanning. The fixation cross subtended a visual angle of about $3.4^{\circ} \times 3.4^{\circ}$, and the pictures subtended a visual angle of about 
$25.3^{\circ} \times 25.3^{\circ}$. All pictures were presented with their center aligned with the screen center.

The design was a block design. There were three experimental conditions, the positive, negative, and the neutral conditions in which pictures of positive, negative, and neutral valence were presented, respectively. Each participant completed four functional runs. Each run contained eight 15-s picture blocks, two positive, two negative, and four neutral, with the affective blocks and neutral blocks alternated. A 15-s fixation block was inserted in between two neighboring picture blocks serving as the resting baseline. The order of positive and negative blocks was counterbalanced across runs and subjects. In each of the 15-s picture blocks, five pictures from the same valence category were presented, each for $3 \mathrm{~s}$ and with zero interstimulus interval. In addition, each run began with a 27-s fixation resting baseline and ended with another 15-s fixation. Each run was thus in total $267 \mathrm{~s}$ long (corresponding to 89 time points with a 3-s TR). Participants were instructed to view the fixation cross or the pictures passively during the whole procedure.

After the imaging session, participants were asked to rate the valence of the pictures they just saw inside the scanner with an 11point scale, where -5 was for most unpleasant and 5 for most pleasant. To avoid fatigue, each participant rated only a subset (1/4) of the pictures from each of the three conditions, which were pseudorandomly intermixed. Across participants, the whole set of picture were rated.

\section{MRI data acquisition}

Imaging was conducted on a 1.5-T Philips Infinion MR system (Philips Medical Systems, Netherlands) in Shantou University Medical College. For each functional run, 89 volumes, each with 23 axial slices, were obtained with a T2*-weighted echo-planar imaging (EPI) sequence $(\mathrm{TR}=3 \mathrm{~s}, \mathrm{TE}=40 \mathrm{~ms}, \mathrm{FOV}=24 \times 24 \mathrm{~cm}$, matrix $=64 \times 64$, flip angle $=90^{\circ}$, slice thickness $=4 \mathrm{~mm}$, gap $\left.=2 \mathrm{~mm}\right)$. A co-planar T1weighted structural volume was obtained with a spin echo (SE) sequence (matrix $=256 \times 256$ ). A high-resolution structural volume was also obtained with a spoiled gradient recalled echo (SPGR) sequence for functional overlay.

\section{Behavioral data analysis}

A $2 \times 3$ repeated-measure analysis of variance (ANOVA) was used for data analysis with participant group (addict, control) and valence (positive, neutral, negative) as factors. Bonferroni correction was used in post hoc analysis. The analysis was performed with SPSS for Windows software (version 17; SPSS Inc., Chicago, IL).

\section{Imaging data analysis}

MRI data were analyzed using the AFNI software package (version: AFNI_2008_07_18_170; Cox, 1996). The first three volumes in each runs were discarded as dummy scans. All functional images were first pre-processed for motion artifact correction, linear drifts removal, temporal smoothing $(0.1 \mathrm{~Hz}$ low-pass filter), and spatial smoothing (5-mm FWHM Gaussian kernel). The imaging data from one addict participant were discarded due to excessive head motion.

Hemodynamic responses for the positive, negative, and neutral conditions were then estimated in each voxel for each participant using deconvolution analysis, with the six motion parameters $(x, y, z$ translations, roll, pitch, and yaw) as covariates to remove residual motion artifacts. The estimated coefficients were scaled to indicate percent signal changes. This generated for each participant three BOLD signal images for the positive, negative, and neutral conditions, respectively, which were then normalized with a transformation to the Talairach space (Talairach and Tournoux, 1988), re-sampled to a voxel size of $2 \times 2 \times 2 \mathrm{~mm}^{3}$.
To assess valence-specific activations, two difference images were computed for each participant by subtracting their normalized signal images of the neutral condition from that of the positive and the negative valence conditions. These difference images were then entered into a random-effect group analysis, which was a $2 \times 2$ ANOVA with participant group (addict vs. control) and valence (positive vs. negative) as factors. The ANOVA was to identify regions of interest (ROIs) across the whole brain in which the BOLD responses were modulated by the group factor, the valence factor, or the interaction of the two. The significance level was set at a voxel-wise threshold of $p<0.005$ and a minimal contiguous volume of $336 \mathrm{~mm}^{3}$, which equals to $p<0.05$ corrected for multiple comparisons (small volume correction in a $7 \times 7 \times 7 \mathrm{~cm}^{3}$ search volume, based on Monte Carlo simulation; Morris et al., 1998). The same difference images were also used in one-sample $t$-tests to make four activation maps (positive and negative in both groups) that were first filtered with a lenient threshold (voxel-wise $p<0.05$ with a minimal contiguous volume of $200 \mathrm{~mm}^{3}$ ) and then combined with a logical "OR" operation to create an inclusive mask (Callicott et al., 2003). The mask was used to limit the ANOVA results to those regions with greater response to either the positive or the negative pictures than to the neutral ones in at least one participant group, which helps to screen out false-positive artifacts due to deactivations.

For each ROI identified above, the valence-related responses (i.e., normalized signal for the positive or the negative conditions minus that for the neutral condition) from all voxels in that ROI were pooled, producing two mean signal values for each participant, one for the positive and one for the negative valence. A $2 \times 2$ ANCOVA (valence by group) was then conducted on these mean values for post hoc confirmation of the response pattern in each ROI, after covarying out age to adjust for cross-group age differences (Volkow et al., 2005). The threshold for the post hoc tests was set at $p<0.05$.

\section{Results}

Stimulus valence ratings

Due to technical reasons, the rating data were lost in two control participants. Shown in Table 1 are the mean rating results, based on 17 addicts and 14 controls. There was a main effect of valence $(F(2,58)=$ 107.1, $p<0.001$ ) with all pair-wise comparisons being significant (Bonferroni corrected $p<0.001)$. The differences remained significant after covarying out age (ANCOVA, $F(2,56)=108.2, p<0.001$ ). The main effect of group and the group $\times$ valence interaction effect were not significant $(F s<1)$.

\section{Imaging results}

Addicts and controls showed similar activation patterns in the neutral vs. rest contrast. The controls demonstrated significant activation in response to affective pictures against resting in the bilateral primary and secondary visual cortices, bilateral amygdala, and bilateral middle prefrontal cortices. Addicts demonstrated significant activation in response to affective pictures relative to fixation in the bilateral primary and secondary visual cortices and bilateral middle prefrontal cortices, with a smaller area of activation in the amygdala (see Supplementary materials for a figure showing brain activations for both participant groups in all three picture conditions relative to the resting baseline.)

Table 1

Mean and standard deviation of valence ratings for the three types of stimuli with an 11 -point scale ( -5 for most unpleasant and 5 for most pleasant).

\begin{tabular}{llll}
\hline & Negative & Neutral & Positive \\
\hline Addicts $(N=17)$ & $-2.4 \pm 1.6$ & $1.3 \pm 1.8$ & $2.8 \pm 1.3$ \\
Control $(N=14)$ & $-1.9 \pm 1.1$ & $1.0 \pm 1.0$ & $2.6 \pm 1.1$ \\
\hline
\end{tabular}




\section{A Main Effect of Group}

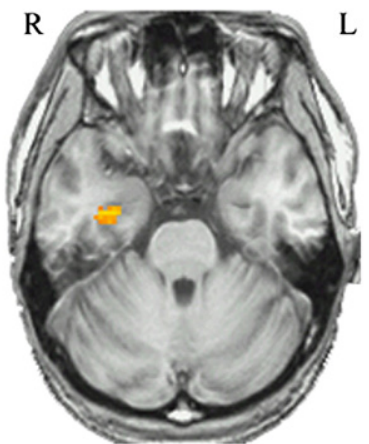

$Z=-22$

B Valence-by-Group Interaction

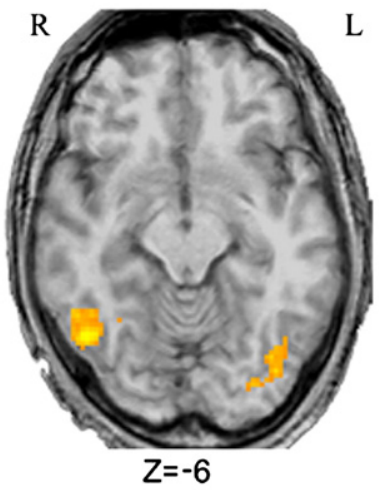

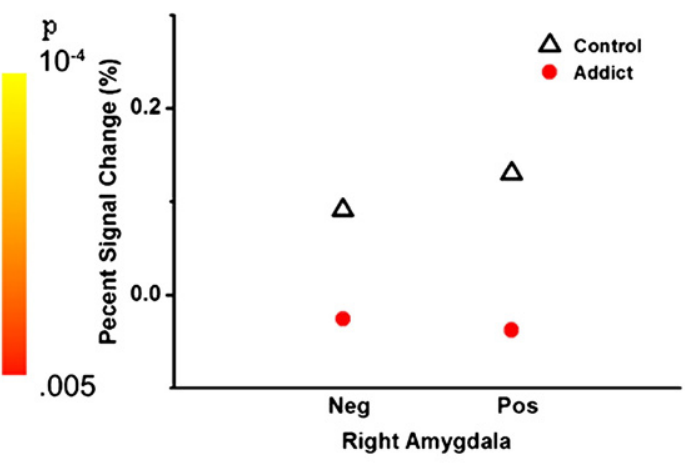

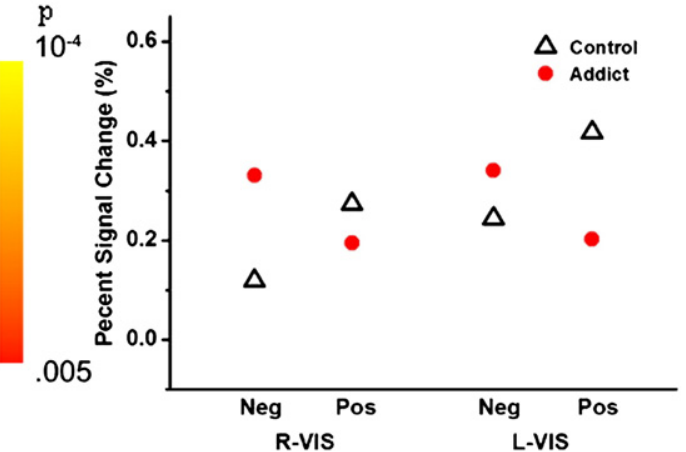

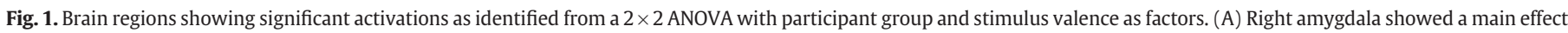

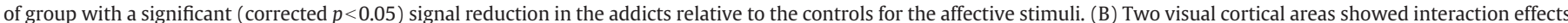

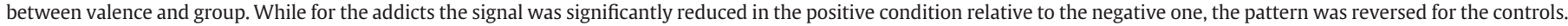

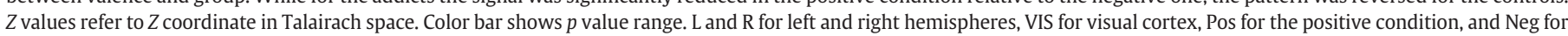
the negative condition.

ANOVA on valence-related brain activities (i.e., differences between responses to affective pictures and that to neutral pictures) revealed a main effect of group in right amygdala as a significant signal reduction in addicts relative to controls $(F(1,30)=18.4$, $p<0.001$; Fig. 1A). There was no brain region showing significant main effect of valence. There was a group $\times$ valence interaction effect in bilateral visual cortices BA 19 and BA $37(F s(1,30)>20.0$, $p s<0.001$; Fig. 1B). The above signal differences in the three ROIs remained significant after covarying out age (ANCOVA, $F s(1,29)>$ $9.8, p s<0.005)$. In bilateral visual cortices, the addicts showed increased brain activation for the negative pictures relative to the positive ones, while the controls showed the opposite pattern ( $p s<0.05$, paired $t$-tests). More details about the activated regions are provided in Table 2.

\section{Table 2}

Summary information for brain regions showing significant affect-related activation for the main effect of group ( 16 addicts vs. 16 controls) and the group $\times$ valence (positive vs. negative) interaction.

\begin{tabular}{|c|c|c|c|c|c|c|}
\hline \multirow[t]{2}{*}{ Anatomic structure } & \multicolumn{3}{|c|}{ Talairach coordinates } & \multirow{2}{*}{$\begin{array}{l}\text { Volume } \\
\left(\mathrm{mm}^{3}\right)\end{array}$} & \multirow{2}{*}{$\begin{array}{l}\text { Peak } F \\
\text { value }\end{array}$} & \multirow[t]{2}{*}{$p^{*}$} \\
\hline & $x$ & $y$ & $z$ & & & \\
\hline \multicolumn{7}{|l|}{ Main effect of group } \\
\hline R amygdale & 30 & -7 & -27 & 336 & 16.1 & 0.05 \\
\hline \multicolumn{7}{|l|}{ Group $\times$ valence interaction } \\
\hline L visual cortex (BA 19/37) & -36 & -75 & -8 & 2336 & 18.9 & 0.001 \\
\hline R visual cortex (BA 19/37) & 42 & -58 & -7 & 2144 & 23.1 & 0.001 \\
\hline
\end{tabular}

L for left hemisphere and $\mathrm{R}$ for right hemisphere.

* Corrected for multiple comparisons with a $7 \times 7 \times 7 \mathrm{~cm}^{3}$ search volume.

\section{Discussions}

The present study revealed several brain regions where the BOLD activity differed significantly across abstinent heroin addicts and normal controls when they passively viewed IPAS pictures of positive, negative, or neutral valence.

One region is right amygdala where the addicts showed decreased brain responses to the affective pictures (pooling over the positive and negative pictures with brain responses to neutral pictures subtracted out) relative to the controls. Although there may be a weaker effect in left amygdala not revealed due to power limit, the results are consistent with literature findings documenting a similar asymmetry of right but not left amygdala volume reduction in addicts (Makris et al., 2004; Matochik et al., 2003).

Amygdala is a key component in the emotion circuitry, important for the experience of emotional feelings and also necessary in triggering emotional states and reactions. For example, amygdala activities are found to correlate with pleasure experience (Keedwell et al., 2005; Zald, 2003). As the imaging literature on addicts with nondrug-related affective materials is limited, there has not been any similar report of amygdala activity alterations. The decreased response in amygdala would imply that addicts have reduced intensity in their emotional feelings towards affective stimuli relative to the normal controls. This is consistent with some behavioral data showing blunted response to affective stimuli in their subjective rating of the stimulus (de Arcos et al., 2005, 2008). As amygdala coordinates the interaction between the behavioral, autonomic, and neuroendocrine systems (Goldstein et al., 1996) and mediates responses to emotional stimuli, this functional abnormality in amygdala 
may also contribute to reduced activation in the neuroendocrine system in heroin addicts found in Gerra et al. (2003).

Wexler et al. (2001) examined neural responses in cocaine addicts to natural affective stimuli. Their results showed that when exposed to sad tapes, abstinent cocaine addicts showed different brain activation dynamics in right hippocampus and right dorsolateral prefrontal cortex (DLPFC) from the controls. Their stimuli were video clips showing an actor verbally describing events related to cocaine use, happy events, or sad events, and their participants had to monitor and report their emotional response with button press. We used static affective pictures and our participants did not have to make any report of their emotional experience. These differences do not allow a direct comparison between the two studies.

Wexler et al. (2001) found a sensory activation reduction during initial video viewing in addicts, relative to the controls, and interpreted it as a generalized alteration in neuro-responsiveness. However, while they did not include a neutral condition and their brain activations were relative to a resting baseline, our results were based on brain response differences between the affective conditions and the neutral conditions. Therefore, the amygdala activity reduction in addicts found here cannot be attributed to a lowered general neural responsiveness (e.g., diminished attention or low task engagement in addicts) but should index genuine differences in processing affective stimuli between addicts and the controls.

Other than amygdala, two visual cortical areas showed greater brain responses to negative stimuli than positive stimuli in addicts, but the pattern was the opposite in controls. These findings in visual cortex were unexpected as previous imaging research on drug addiction mostly focuses on the limbic system and the frontal cortex. However, there is a literature in the normal population documenting valence effects in visual cortical activations (Lang et al., 1998b; Phan et al., 2002; Sabatinelli et al., 2004; Herrmann et al., 2008). Using different imaging methods including fMRI, ERP, and near-infrared spectroscopy (NIRS), researchers showed that processing emotional visual stimuli leads to increased activations of the occipital cortex compared with neutral stimuli. One particularly relevant study is by Sabatinelli et al. (2004) who demonstrated with fMRI a gender difference where male participants showed greater visual activity to pleasant stimuli than to aversive ones but females showed the opposite pattern. The normal controls in the present study were all male, and their results mimicked that of the normal male participants in Sabatinelli et al. study. Therefore, in light of the Sabatinelli et al. study, the addicts' cortical activation observed here can be characterized as either reduced neural response to the positive stimuli or heightened response to the negative stimul, or a combination of the two.

Emotional modulation of visual cortical activity may functionally result from a natural bias in selective attention to stimuli of evolutionary importance (Lang et al., 1998b), anatomically implemented as distant influences on visual cortex from brain structures outside the visual system (Vuilleumier et al., 2004). From this perspective, reduced response to positive stimuli in visual cortical areas, unlike that in amygdala, may not directly index blunted emotional experience but reflect abnormal modulation from the altered emotion circuitry due to drug use. The increased response to the negative stimulus may be understood in a similar way.

Briefly, our results showed that neural response to affective stimuli in addicts is more complex than a general reduction or heightening-while reduced responses to both positive and negative valence were found in some regions, reduced responses to positive stimuli but enhanced responses to negative stimuli were found in other regions.

One limitation of the present study is that, although the patient participants were hospitalized similar to those chronic psychiatric patients and free to take activity in the clinic, they were isolated from the society to prevent drug access. Long hospitalization will inevitably lead to some stress to make them different from patients who are free. So the present results need to be cross-validated by further studies with out-patients.

As reviewed in the Introduction, behavioral studies directly asking addict participants to self-report their subjective experience of affective stimuli show mixed pattern of results, such as blunted, heightened, or unchanged rating for either positive or negative or both positive and negative stimuli (e.g., de Arcos et al., 2005, 2008; Dawkins et al., 2007). In the present study, participants rated the materials after the scan and showed comparable ratings as the controls. Apparently, it remains unclear how to relate abnormal brain response patterns in addicts to their altered emotional experience based on subjective report. It is possible that self-report may only reveal a global measure of emotional feelings and the mapping between this single measure and activities in multiple brain regions can be complex. For example, self-report may not reveal subconscious activation detectable with brain imaging methods. To make things more complicated, self-report and subjective experience may fall on different dimensions of emotion such as emotional judgment and emotional perception (Grimm et al., 2006; Heinzel et al., 2005). More imaging research are therefore called for to examine the processing of everyday affective materials in addicts for more objective and richer information in understanding disruptions of their emotion processing mechanism. Such research would also help to identify effective neural markers for the monitoring of treatment effects and for the development of effective interventions.

\section{Acknowledgments}

This research was supported by the National Nature Science Foundation of China (No. 30200068, 30670598, 30670702, 30700235, 30420120048), the Fok Ying Tung Education Foundation (No. 91038), and the China Postdoctoral Science Foundation (No. 20070410845). We thank Linfa Wu, Genglong Zhen, and Shaoxin Chen for assistance in carrying out the study. We thank an anonymous reviewer for his/ her insightful and valuable comments on earlier versions of the paper. We declare that there are no conflicts of interest.

\section{Appendix A. Supplementary data}

Supplementary data associated with this article can be found, in the online version, at doi:10.1016/j.neuroimage.2009.08.020.

\section{References}

Baker, T.B., Piper, M.E., McCarthy, D.E., Majeskie, M.R., Fiore, M.C., 2004. Addiction motivation reformulated: an affective processing model of negative reinforcement. Psychol. Rev. 111, 33-51.

Baler, R.D., Volkow, N.D., 2006. Drug addiction: the neurobiology of disrupted selfcontrol. Trends Mol. Med. 12, 559-566.

Bechara, A., Damasio, H., 2002. Decision-making and addiction (part I): impaired activation of somatic states in substance dependent individuals when pondering decisions with negative future consequences. Neuropsychologia 40, 1675-1689.

Bjork, J.M., Smith, A.R., Hommer, D.W., 2008. Striatal sensitivity to reward deliveries and omissions in substance dependent patients. Neuroimage 42, 1609-1621.

Callicott, J.H., Mattay, V.S., Verchinski, B.A., Marenco, S., Egan, M.F., Weinberger, D.R., 2003. Complexity of prefrontal cortical dysfunction in schizophrenia: more than up or down. Am. J. Psychiatry 160, 2209-2215.

Compton, R.J., Banich, M.T., Mohanty, A., Milham, M.P., Herrington, J., Miller, G.A., Scalf, P.E., Webb, A., Heller, W., 2003. Paying attention to emotion: an fMRI investigation of cognitive and emotional Stroop tasks. Cogn. Affect. Behav. Neurosci. 3, 81-96.

Cox, R.W., 1996. AFNI: software for analysis and visualization of functional magnetic resonance neuroimages. Comput. Biomed. Res. 29, 162-173.

Dawkins, L., Acaster, S., Powell, J.H., 2007. The effects of smoking and abstinence on experience of happiness and sadness in response to positively valenced, negatively valenced, and neutral film clips. Addict. Behav. 32, 425-431.

de Arcos, F.A., Verdejo-Garcia, A., Ceverino, A., Montanez-Pareja, M., Lopez-Juarez, E., Sanchez-Barrera, M., Lopez-Jimenez, A., Perez-Garcia, M., 2008. Dysregulation of emotional response in current and abstinent heroin users: negative heightening and positive blunting. Psychopharmacology (Berl) 198, 159-166.

de Arcos, F.A., Verdejo-Garcia, A., Peralta-Ramirez, M.I., Sanchez-Barrera, M., PerezGarcia, M., 2005. Experience of emotions in substance abusers exposed to images 
containing neutral, positive, and negative affective stimuli. Drug Alcohol Depend. 78, 159-167.

Garavan, H., Pankiewicz, J., Bloom, A., Cho, J.K., Sperry, L., Ross, T.J., Salmeron, B.J., Risinger, R., Kelley, D., Stein, E.A., 2000. Cue-induced cocaine craving: neuroanatomical specificity for drug users and drug stimuli. Am. J. Psychiatry 157, 1789-1798.

George, M.S., Anton, R.F., Bloomer, C., Teneback, C., Drobes, D.J., Lorberbaum, J.P., Nahas, Z., Vincent, D.J., 2001. Activation of prefrontal cortex and anterior thalamus in alcoholic subjects on exposure to alcohol-specific cues. Arch. Gen. Psychiatry 58, 345-352.

Gerra, G., Baldaro, B., Zaimovic, A., Moi, G., Bussandri, M., Raggi, M.A., Brambilla, F., 2003. Neuroendocrine responses to experimentally-induced emotions among abstinent opioid-dependent subjects. Drug Alcohol Depend. 71, 25-35.

Goldstein, L.E., Rasmusson, A.M., Bunney, B.S., Roth, R.H., 1996. Role of the amygdala in the coordination of behavioral, neuroendocrine, and prefrontal cortical monoamine responses to psychological stress in the rat. J. Neurosci. 16, 4787-4798.

Goldstein, R.Z., Alia-Klein, N., Tomasi, D., Zhang, L., Cottone, L.A., Maloney, T., Telang, F., Caparelli, E.C., Chang, L., Ernst, T., Samaras, D., Squires, N.K., Volkow, N.D., 2007. Is decreased prefrontal cortical sensitivity to monetary reward associated with impaired motivation and self-control in cocaine addiction? Am. J. Psychiatry 164, 43-51.

Grant, S., London, E.D., Newlin, D.B., Villemagne, V.L., Liu, X., Contoreggi, C., Phillips, R.L., Kimes, A.S., Margolin, A., 1996. Activation of memory circuits during cue-elicited cocaine craving. Proc. Natl. Acad. Sci. U. S. A. 93, 12040-12045.

Grimm, S., Schmidt, C.F., Bermpohl, F., Heinzel, A., Dahlem, Y., Wyss, M., Hell, D., Boesiger, P., Boeker, H., Northoff, G., 2006. Segregated neural representation of distinct emotion dimensions in the prefrontal cortex-an fMRI study. Neuroimage 30, 325-340.

Heinzel, A., Bermpohl, F., Niese, R., Pfennig, A., Pascual-Leone, A., Schlaug, G., Northoff, G., 2005. How do we modulate our emotions? Parametric fMRI reveals cortical midline structures as regions specifically involved in the processing of emotional valences. Cogn. Brain Res. 25, 348-358.

Herrmann, M.J., Huter, T., Plichta, M.M., Ehlis, A.C., Alpers, G.W., Muhlberger, A., Fallgatter, A.J., 2008. Enhancement of activity of the primary visual cortex during processing of emotional stimuli as measured with event-related functional near-infrared spectroscopy and event-related potentials. Hum. Brain Mapp. 29, 28-35.

Keedwell, P.A., Andrew, C., Williams, S.C., Brammer, M.J., Phillips, M.L., 2005. The neural correlates of anhedonia in major depressive disorder. Biol. Psychiatry 58, 843-853.

Lang, P.J., Bradley, M.M., Cuthbert, B.N., 1998a. International Affective Picture System (IAPS): Technical Manual and Affective Ratings. Center for Research in Psychophysiology, University of Florida, Gainesvillel, FL.
Lang, P.J., Bradley, M.M., Fitzsimmons, J.R., Cuthbert, B.N., Scott, J.D., Moulder, B., Nangia, V., 1998b. Emotional arousal and activation of the visual cortex: an fMRI analysis. Psychophysiology 35, 199-210.

Makris, N., Gasic, G.P., Seidman, L.J., Goldstein, J.M., Gastfriend, D.R., Elman, I., Albaugh, M.D., Hodge, S.M., Ziegler, D.A., Sheahan, F.S., Caviness Jr., V.S., Tsuang, M.T. Kennedy, D.N., Hyman, S.E., Rosen, B.R., Breiter, H.C., 2004. Decreased absolute amygdala volume in cocaine addicts. Neuron 44, 729-740.

Matochik, JA, London, E.D., Eldreth, D.A., Cadet, J.L., Bolla, K.I, 2003. Frontal cortical tissue composition in abstinent cocaine abusers: a magnetic resonance imaging study. Neuroimage 19, 1095-1102.

Morris, J.S., Friston, K.J., Buchel, C., Frith, C.D., Young, A.W., Calder, A.J., Dolan, R.J., 1998 A neuromodulatory role for the human amygdala in processing emotional facial expressions. Brain 121, 47-57.

Phan, K.L., Wager, T., Taylor, S.F., Liberzon, I., 2002. Functional neuroanatomy of emotion: a meta-analysis of emotion activation studies in PET and fMRI. Neuroimage 16 331-348.

Russell, J.A., Barrett, L.F., 1999. Core affect, prototypical emotional episodes, and other things called emotion: dissecting the elephant. J. Pers. Soc. Psychol. 76, 805-819.

Sabatinelli, D., Flaisch, T., Bradley, M.M., Fitzsimmons, J.R., Lang, P.J., 2004. Affective picture perception: gender differences in visual cortex? Neuroreport 15, 1109-1112.

Talairach, J., Tournoux, P., 1988. Co-planar Stereotaxic Atlas of the Human Brain. Georg Thieme Verlag, Stuttgart.

Volkow, N.D., Fowler, J.S., 2000. Addiction, a disease of compulsion and drive: involvement of the orbitofrontal cortex. Cereb. Cortex 10, 318-325.

Volkow, N.D., Wang, G.J., Ma, Y., Fowler, J.S., Wong, C., Ding, Y.S., Hitzemann, R. Swanson, J.M., Kalivas, P., 2005. Activation of orbital and medial prefrontal cortex by methylphenidate in cocaine-addicted subjects but not in controls: relevance to addiction. J. Neurosci. 25, 3932-3939.

Vuilleumier, P., Richardson, M.P., Armony, J.L., Driver, J., Dolan, R.J., 2004. Distant influences of amygdala lesion on visual cortical activation during emotional face processing. Nat. Neurosci. 7, 1271-1278.

Washton, A.M., Stone-Washton, N., 1993. Outpatient treatment of cocaine and crack addiction: a clinical perspective. NIDA Res. Monogr. 135, 15-30.

Wexler, B.E., Gottschalk, C.H., Fulbright, R.K., Prohovnik, I., Lacadie, C.M., Rounsaville, B.J., Gore, J.C., 2001. Functional magnetic resonance imaging of cocaine craving. Am. J. Psychiatry 158, 86-95.

Xiao, Z.W., Lee, T., Zhang, J.X., Wu, Q.L., Wu, R.H., Weng, X.C., Hu, X.P., 2006. Thirsty heroin addicts show different fMRI activations when exposed to water-related and drug-related cues. Drug Alcohol Depend. 83, 157-162.

Zald, D.H., 2003. The human amygdala and the emotional evaluation of sensory stimuli. Brain Res. Brain Res. Rev. 41, 88-123. 